\title{
Ceramic Types and Varieties
}

(in alphabetical order)

Aac Red-on-buff: Aac Variety . . . . . . . . . . . . . . . . . . . . . . . . . . . . 22

Accordian Incised: Design-incised Variety (Cauac Complex) . . . . . . . . . . . . . . . 134

Accordian Incised: Design-incised Variety (Cimi Complex) . . . . . . . . . . . . . . . . . . 174

Accordian Incised: Simple-incised Variety (Cauac Complex) . . . . . . . . . . . . . . . . . . 134

Accordian Incised: Simple-incised Variety (Chuen Complex) $\ldots$. . . . . . . . . . . . . . . . . 98

Accordian Incised: Simple-incised Variety (Cimi Complex) . . . . . . . . . . . . . . . . . 174

Achiotes Unslipped: Achiotes Variety (Cauac Complex) . . . . . . . . . . . . . . . . . . . . . 120

Achiotes Unslipped: Achiotes Variety (Chuen Complex) ․ . . . . . . . . . . . . . . . . . 85

Achiotes Unslipped: Achiotes Variety (Cimi Complex) . . . . . . . . . . . . . . . . . . . 162

Achiotes Unslipped: Achiotes Variety (Tzec Complex) … . . . . . . . . . . . . . . . .52

Achote Black: Achote Variety (Eznab Complex) . . . . . . . . . . . . . . . . . . . . 350



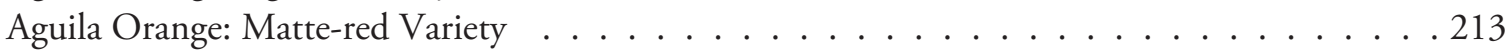

Ahchab Red-on-buff: Ahchab Variety . . . . . . . . . . . . . . . . . . . . . . . . . . . . . 89



Ainil Orange: Ainil Variety . . . . . . . . . . . . . . . . . . . . . . . 27

Alta Mira Fluted: Alta Mira Variety (Cauac Complex) . . . . . . . . . . . . . . . . . . . . . . . 126

Alta Mira Fluted: Alta Mira Variety (Cimi Complex) . . . . . . . . . . . . . . . . . 167

Altar Orange: Altar Variety . . . . . . . . . . . . . . . . . . . . . . . . 358

Amil Chamfered: Amil Variety . . . . . . . . . . . . . . . . . . . . . 56

Baadz Tan: Baadz Variety . . . . . . . . . . . . . . . . . . . . . 30

Baclam Red-orange: Ahtau Variety . . . . . . . . . . . . . . . . . . . . . . . . . .58

Baclam Red-orange: Baclam Variety (Cauac Complex) . . . . . . . . . . . . . . . . . . . 131

Baclam Red-orange: Baclam Variety (Chuen Complex) . . . . . . . . . . . . . . . . . . . . . 95

Baclam Red-orange: Baclam Variety (Cimi Complex) . . . . . . . . . . . . . . . . . . 171

Balanza Black: Balanza Variety . . . . . . . . . . . . . . . . . . . . . . . . . 214

Bechh Incised: Design-incised Variety. . . . . . . . . . . . . . . . . . . . . . . . 29

Bechh Incised: Simple-incised Variety. . . . . . . . . . . . . . . . . . . . . . . 29

Bil White: Bil Variety . . . . . . . . . . . . . . . . . . . . . . . . . 30



Boolay Brown: Boolay Variety. . . . . . . . . . . . . . . . . . . . . 28

Boolim Red-on-cream: Boolim Variety . . . . . . . . . . . . . . . . . . . . . . . . 59

Boxcay Brown: Boxcay Variety (Cauac Complex) . . . . . . . . . . . . . . . . . . . . . 132

Boxcay Brown: Boxcay Variety (Chuen Complex) … . . . . . . . . . . . . . . . 96 
Boxcay Brown: Boxcay Variety (Cimi Complex) ～. . . . . . . . . . . . . . . . . . . . . 172

Cabcoh Striated: Cabcoh Variety . . . . . . . . . . . . . . . . . . . . . . . . . . . . 19

Cabro Red: Cabro Variety (Cauac Complex) ․ . . . . . . . . . . . . . . . . . 128

Cabro Red: Cabro Variety (Cimi Complex) . . . . . . . . . . . . . . . . . . . . 168

Calam Buff: Calam Variety . . . . . . . . . . . . . . . . . . . . . . . . . . . . . . . . . .

Caldero Buff Polychrome: Caldero Variety . . . . . . . . . . . . . . . . . . . . . . . 221



Cambio Unslipped: Cambio Variety (Ik Complex) . . . . . . . . . . . . . . . . . . . . . . 262

Cambio Unslipped: Cambio Variety (Imix Complex) . . . . . . . . . . . . . . . . . . . . . . 299

Cameron Incised: Cameron Variety (Eznab Complex) . . . . . . . . . . . . . . . . . . . 348

Cameron Incised: Cameron Variety (Ik Complex) ～. . . . . . . . . . . . . . . . . . 266

Cameron Incised: Cameron Variety (Imix Complex) … . . . . . . . . . . . . . . 304

Canhel Unslipped: Canhel Variety . . . . . . . . . . . . . . . . . . . . . . . 17

Caramba Red-on-Orange: Chic Variety (Cauac Complex) . . . . . . . . . . . . . . . . . . . . 134

Caramba Red-on-orange: Chic Variety (Cimi Complex) . . . . . . . . . . . . . . . . . . . 174

Cay Incised: Design-incised Variety (Cauac Complex) . . . . . . . . . . . . . . . . . . . . . . . . . . . . . .

Cay Incised: Design-incised Variety $($ Chuen Complex) $\ldots \ldots$. . . . . . . . . . . . . . . . . . . . . . . . . . . . .

Cay Incised: Design-incised Variety (Cimi Complex) . . . . . . . . . . . . . . . . . . . . 172

Cay Incised: Simple-incised Variety (Cauac Complex) . . . . . . . . . . . . . . . . . . . . . . . . . . . . . . . . . . .

Cay Incised: Simple-incised Variety $($ Chuen Complex) $\ldots \ldots$. . . . . . . . . . . . . . . . . . . . . . . . . . . .

Cay Incised: Simple-incised Variety (Cimi Complex) . . . . . . . . . . . . . . . . . . . . . . 172

Cayetano Trichrome: Cayetano Variety ． . . . . . . . . . . . . . . . . . . . 177

Cayetano Trichrome: Xnuk Variety ． . . . . . . . . . . . . . . . . . . . 178

Chantuori Black-on-orange: Chantuori Variety (Eznab Complex) . . . . . . . . . . . . . . . 354

Chantuori Black-on-orange: Chantuori Variety (Imix Complex) . . . . . . . . . . . . . . . . . . . . . . 270

Chilar Fluted: Chilar Variety (Ik Complex) . . . . . . . . . . . . . . . . . . . . . . . . 270

Chilar Fluted: Chilar Variety (Imix Complex) . . . . . . . . . . . . . . . . . . . . 308

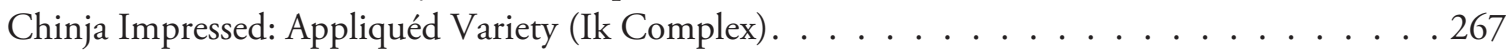

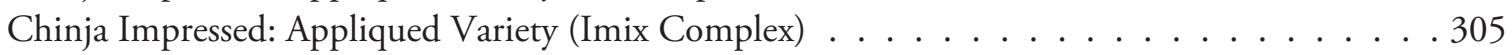



Chinja Impressed: Chinja Variety (Ik Complex) ～. . . . . . . . . . . . . . . . . . . 367

Chinja Impressed: Chinja Variety (Imix Complex) ～. . . . . . . . . . . . . . . . . 304

Chinos Black-on-cream: Chinos Variety (Eznab Complex) . . . . . . . . . . . . . . . . 356

Chinos Black-on-cream: Chinos Variety (Imix Complex) . . . . . . . . . . . . . . . . . . 313



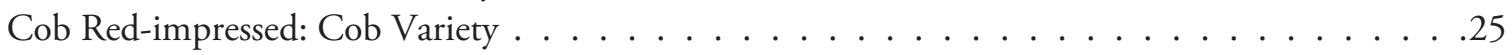



Correlo Incised-dichrome: Correlo Variety (Cauac Complex) . . . . . . . . . . . . . . . . . . . 129

Correlo Incised-dichrome: Correlo Variety (Cimi Complex) . . . . . . . . . . . . . . . . . . . 169

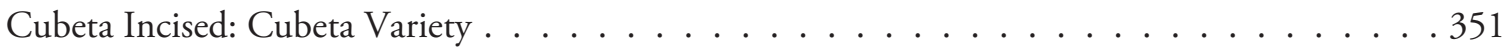

Delirio Plano-relief: Delirio Variety ． . . . . . . . . . . . . . . . . . . . 217



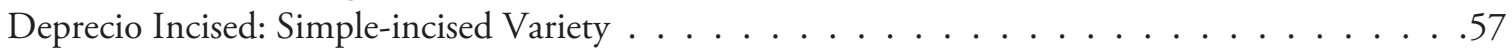

Desquite Red-on-orange: Desquite Variety . . . . . . . . . . . . . . . . . . . . . . . . . 272

Dos Arroyos Orange Polychrome: Dos Arroyos Variety . . . . . . . . . . . . . . . . . . . 219

Encanto Striated: Encanto Variety (Eznab Complex) . . . . . . . . . . . . . . . . . 345 
Encanto Striated: Encanto Variety (Ik Complex) . . . . . . . . . . . . . . . . . . . . . . . 264

Encanto Striated: Encanto Variety (Imix Complex) . . . . . . . . . . . . . . . . . . . . . . 302

Fama Buff: Fama Variety . . . . . . . . . . . . . . . . . . . . . . . . . . . . . . . . . . 219

Flor Cream: Flor Variety (Cauac Complex) ․ . . . . . . . . . . . . . . . . . . . . 133

Flor Cream: Flor Variety (Chuen Complex) $\ldots \ldots$. . . . . . . . . . . . . . . . . . 97

Flor Cream: Variety (Cimi Complex) . . . . . . . . . . . . . . . . . . . . . . . . . . . . . . . . . . . . . . . .

Guitara Incised: Design-incised Variety . . . . . . . . . . . . . . . . . . . . . . . . . . . . . . . . . . . . .

Guitara Incised: Simple-incised Variety . . . . . . . . . . . . . . . . . . . 55

Haleb Composite: Haleb Variety . . . . . . . . . . . . . . . . . . . . . . 31

Hiabon Punctated: Hiabon Variety (Cauac Complex) ․ . . . . . . . . . . . . . . . . . 127

Hiabon Punctated: Hiabon Variety (Cimi Complex) . . . . . . . . . . . . . . . . . . . . . 167

Jama Red Polychrome: Jama Variety (Ik Complex) . . . . . . . . . . . . . . . . . . . . . . . . 273

Japon Resist: Japon Variety . . . . . . . . . . . . . . . . . . . . . . . . . . . . 223

Jato Black-on-gray: Jato Variety . . . . . . . . . . . . . . . . . . . . . . . . . . . 357

Joventud Red: Ahcax Variety . . . . . . . . . . . . . . . . . . . . . . . . . . . . 54

Kanalcan Gouged-incised: Kanalcan Variety . . . . . . . . . . . . . . . . . . . . 315

Kau Incised: Kau Variety . . . . . . . . . . . . . . . . . . . . . . . . . 268

Kokob Carved: Kokob Variety . . . . . . . . . . . . . . . . . . . . . . 265

Lagartos Punctated: Lagartos Variety (Cauac Complex) . . . . . . . . . . . . . . . . . . 127

Lagartos Punctated: Lagartos Variety (Chuen Complex) ․ . . . . . . . . . . . . . . . . 92

Lagartos Punctated: Lagartos Variety (Cimi Complex) . . . . . . . . . . . . . . . . . . 167

Laguna Verde Incised: Design-incised Variety (Cauac Complex) . . . . . . . . . . . . . . . 125

Laguna Verde Incised: Design-incised Variety (Chuen Complex) . . . . . . . . . . . . . . . . .92

Laguna Verde Incised: Design-incised Variety (Cimi Complex) . . . . . . . . . . . . . . 165

Laguna Verde Incised: Simple-incised Variety (Cauac Complex) . . . . . . . . . . . . . . . . 125

Laguna Verde Incised: Simple-incised Variety (Chuen Complex) . . . . . . . . . . . . . . . . . .92 . . . . . . .

Laguna Verde Incised: Simple-incised Variety (Cimi Complex) . . . . . . . . . . . . . . . . . 165

Laguna Verde Incised: Usulutan-style Variety (Cauac Complex) . . . . . . . . . . . . . . . . 125

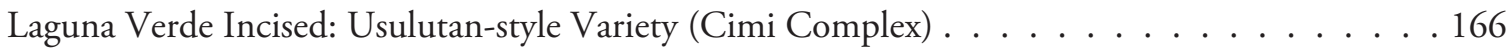

Lechugal Incised: Design-incised Variety (Cauac Complex) . . . . . . . . . . . . . . . . 131

Lechugal Incised: Design-incised Variety (Chuen Complex) $\ldots$. . . . . . . . . . . . . . .95

Lechugal Incised: Design-incised Variety (Cimi Complex) . . . . . . . . . . . . . . . . 171

Lechugal Incised: Simple-incised Variety (Cauac Complex) . . . . . . . . . . . . . . . . 131

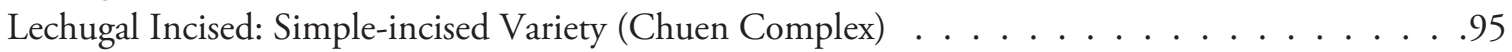

Lechugal Incised: Simple-incised Variety (Cimi Complex) ． . . . . . . . . . . . . . . . 171

Lucha Incised: Lucha Variety . . . . . . . . . . . . . . . . . . . . . . 216

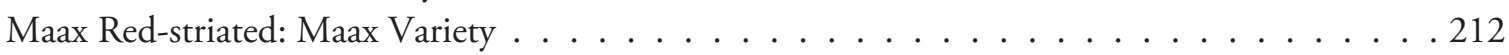

Maquina Brown: Maquina Variety (Eznab Complex) . . . . . . . . . . . . . . . . . . . . 352

Maquina Brown: Maquina Variety (Ik Complex) . . . . . . . . . . . . . . . . . . . . . . . . 269

Maquina Brown: Maquina Variety (Imix Complex) . . . . . . . . . . . . . . . . . . 306

Metapa Trichrome: Itsul Variety (Cauac Complex) . . . . . . . . . . . . . . . . . . . 136

Metapa Trichrome: Itsul Variety (Cimi Complex) ～. . . . . . . . . . . . . . . . . . . 176

Mex Composite: Mex Variety . . . . . . . . . . . . . . . . . . . . . . . . . . . 314

Moc Orange Polychrome: Moc Variety . . . . . . . . . . . . . . . . . . . . . . . . 223

Mojara Orange Polychrome: Mojara Variety ․ . . . . . . . . . . . . . . . . . 178

Morfin Unslipped: Morfin Variety (Cauac Complex) . . . . . . . . . . . . . . . . 123 
Morfin Unslipped: Morfin Variety (Cimi Complex) ． . . . . . . . . . . . . . . . . . . . . 164

Mut Dichrome: Mut Variety (Cauac Complex) . . . . . . . . . . . . . . . . . . . . . . . . . . 129

Mut Dichrome: Mut Variety (Cimi Complex) … . . . . . . . . . . . . . . . . . . 164

Naranjal Red-on-cream: Naranjal Variety (Eznab Complex) . . . . . . . . . . . . . . . . 356

Naranjal Red-on-cream: Naranjal Variety (Imix Complex) . . . . . . . . . . . . . . . . . . . 313

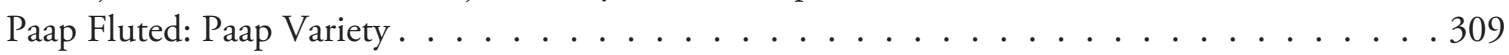

Pabellon Molded-carved: Pabellon Variety . . . . . . . . . . . . . . . . . . . . . . . . . 359

Paixban Buff Polychrome: Paixban Variety . . . . . . . . . . . . . . . . . . . 314

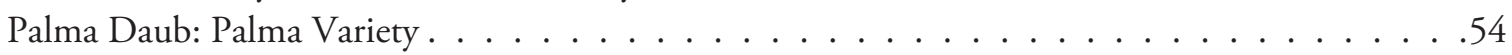

Palmar Orange Polychrome: Palmar Variety (Eznab Complex) . . . . . . . . . . . . . . . 353

Palmar Orange Polychrome: Palmar Variety (Imix Complex) . . . . . . . . . . . . . . . . . . . 309

Palmar Orange Polychrome: Red-bar Variety . . . . . . . . . . . . . . . . . . . . . . . 310

Pantano Impressed: Stamped Variety ～. . . . . . . . . . . . . . . . . . . . . . . . . . 349

Paradero Fluted: Paradero Variety. . . . . . . . . . . . . . . . . . . . . . . . . . . . 217

Pepet Incised: Pepet Variety. . . . . . . . . . . . . . . . . . . . . . . . . . . . 352

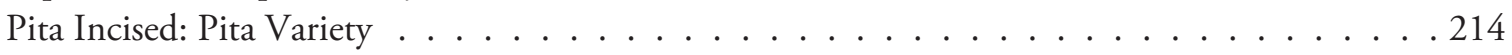

Pital Cream: Unspecified Variety . . . . . . . . . . . . . . . . . . . . . . . . . . . . 59

Polvero Black: Polvero Variety (Cauac Complex) . . . . . . . . . . . . . . . . . . . . 130

Polvero Black: Polvero Variety (Chuen Complex) . . . . . . . . . . . . . . . . . . . . 94

Polvero Black: Polvero Variety (Cimi Complex). . . . . . . . . . . . . . . . . . . . . . . 170

Positas Modeled: Positas Variety ． . . . . . . . . . . . . . . . . . . . . . . . . . . 218

Pucte Brown: Pucte Variety . . . . . . . . . . . . . . . . . . . . . . . . . . 218

Quintal Unslipped: Quintal Variety. . . . . . . . . . . . . . . . . . . . . . . . . . . . 209

Reforma Incised: Reforma Variety. . . . . . . . . . . . . . . . . . . . . . . . . . 61

Repasto Black-on-red: Repasto Variety (Cauac Complex) . . . . . . . . . . . . . . . . . . . . . 127

Repasto Black-on-red: Repasto Variety (Chuen Complex) ． . . . . . . . . . . . . . . . . . 93

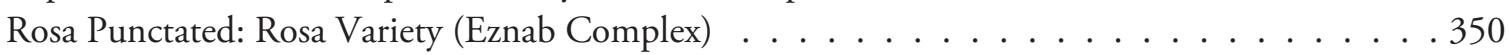

Rosa Punctated: Rosa Variety (Ik Complex) . . . . . . . . . . . . . . . . . . . . . . . . 268

Rosa Punctated: Rosa Variety (Imix Complex) . . . . . . . . . . . . . . . . . . . . . . . 305



Sacluc Black-on-orange: Sis Variety (Cimi Complex) . . . . . . . . . . . . . . . . . . . . . 175

Sacluc Black-on-orange: Xux Variety . . . . . . . . . . . . . . . . . . . . . 176

Sahcaba Modeled-carved: Sahcaba Variety . . . . . . . . . . . . . . . . . . . 358

Salada Fluted: Salada Variety ． . . . . . . . . . . . . . . . . . . . . . . . . 306

San Bartolo Red-on-buff: San Bartolo Variety ． . . . . . . . . . . . . . . . . . . . 222

San Blas Red-on-orange: San Blas Variety ． . . . . . . . . . . . . . . . . . . . . . 221

San Clemente Gouged-incised: San Clemente Variety. . . . . . . . . . . . . . . . . . . . . . . . . . . . . . . . . . . . . .



Sapote Striated: Sapote Variety (Cauac Complex) . . . . . . . . . . . . . . . . . . . . . . . . . 122



Sapote Striated: Sapote Variety (Cimi Complex) ～. . . . . . . . . . . . . . . . . . . . . 163

Savana Orange: Savana Variety . . . . . . . . . . . . . . . . . . . . . . . . . . 60

Saxche Orange Polychrome: Saxche Variety . . . . . . . . . . . . . . . . . . . 271

Sibal Buff Polychrome: Sibal Variety . . . . . . . . . . . . . . . . . . . . . . . . . . 273

Sierra Red: Ahuacan Variety. . . . . . . . . . . . . . . . . . . . . . . . . . . . . . . . 89

Sierra Red: Sierra Variety (Cauac Complex) . . . . . . . . . . . . . . . . . . . 124 
Sierra Red: Sierra Variety (Chuen Complex) ～. . . . . . . . . . . . . . . . . . . . . .90

Sierra Red: Sierra Variety (Cimi Complex) … . . . . . . . . . . . . . . . . . . . 164

Society Hall Red: Society Hall Variety ～. . . . . . . . . . . . . . . . . . . . . . . .91

Tinaja Red: Tinaja Variety (Eznab Complex) . . . . . . . . . . . . . . . . . . . . . . . . 347



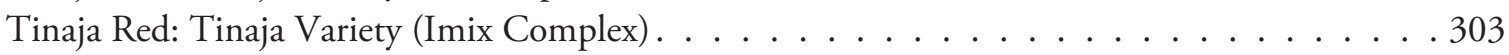

Toh Brown: Toh Variety . . . . . . . . . . . . . . . . . . . . . . . . 352

Tott Incised: Tott Variety . . . . . . . . . . . . . . . . . . . . . . . . 353

Trapiche Incised: Trapiche Variety . . . . . . . . . . . . . . . . . . . . . . . 359

Triunfo Striated: Triunfo Variety . . . . . . . . . . . . . . . . . . . . . . 211

Tumba Black-on-orange: Tumba Variety . . . . . . . . . . . . . . . . . . . . . . . . . . . 359

Uacho Black-on-orange: Uacho Variety . . . . . . . . . . . . . . . . . . . . . . . . . 272

Ucum Unslipped: Ucum Variety (Ik Complex) . . . . . . . . . . . . . . . . . . . . . . 264

Ucum Unslipped: Ucum Variety (Imix Complex) . . . . . . . . . . . . . . . . . . . . . . . 302

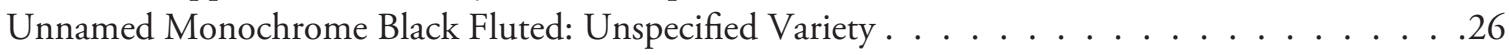

Unnamed Monochrome Black Incised: Fine Inclusions Variety . . . . . . . . . . . . . . . . . . . . 26

Unnamed Monochrome Black: Fine Inclusions Variety . . . . . . . . . . . . . . . . . . . . . . . . . . . . .

Unnamed Monochrome Red Fluted: Unspecified Variety. . . . . . . . . . . . . . . . . . . . . . . . . . . .

Unnamed Monochrome Red Incised: Fine Inclusions Variety. . . . . . . . . . . . . . . . 23

Unnamed Monochrome Red Incised: Yellow-paste Variety . . . . . . . . . . . . . . . . . 24

Unnamed Monochrome Red: Fine Inclusions Variety. . . . . . . . . . . . . . . . . . . . . 22

Unnamed Monochrome Red: Yellow-paste Variety . . . . . . . . . . . . . . . . . . . . . . . . . . . . . . . . . . .

Urita Gouged-incised: Urita Variety ． . . . . . . . . . . . . . . . . . . . 216

Uz Buff: Uz Variety (Ik Complex). . . . . . . . . . . . . . . . . . . . . . . . . . . . 271

Uz Buff: Uz Variety (Imix Complex) ․ . . . . . . . . . . . . . . . . . . . . . . . 308

Veracal Orange: Veracal Variety (Ik Complex) ～. . . . . . . . . . . . . . . . . . . 268

Veracal Orange: Veracal Variety (Imix Complex) . . . . . . . . . . . . . . . . . . 306

Xcuican Incised-dichrome: Xcuican Variety ～. . . . . . . . . . . . . . . . . .93

Xik Double-slipped Orange: Xik Variety … . . . . . . . . . . . . . . . . . . 98

Xkili Fluted: Xkili Variety. . . . . . . . . . . . . . . . . . . . . . . . . . . . 29

Xpokol Incised: Design-incised Variety ． . . . . . . . . . . . . . . . . . . . . . 28

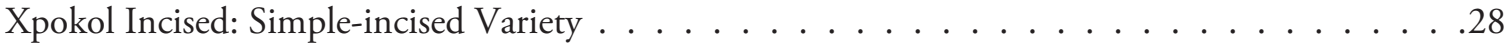

Xtabcab Incised: Design-incised Variety (Cauac Complex) . . . . . . . . . . . . . . . 133

Xtabcab Incised: Design-incised Variety (Chuen Complex) . . . . . . . . . . . . . . . . . . .97

Xtabcab Incised: Design-incised Variety (Cimi Complex) . . . . . . . . . . . . . . . . . . . 173

Xtabcab Incised: Simple-incised Variety (Cauac Complex) . . . . . . . . . . . . . . . . . 133

Xtabcab Incised: Simple-incised Variety (Chuen Complex) . . . . . . . . . . . . . . . . . . . . . . . . . . . . . .

Xtabcab Incised: Simple-incised Variety (Cimi Complex) . . . . . . . . . . . . . . . . . 173

Xtoyil Fluted: Xtoyil Variety . . . . . . . . . . . . . . . . . . . . . . . . . 28

Yaloche Cream Polychrome: Yaloche Variety . . . . . . . . . . . . . . . . . . . 222

Yuhactal Black-on-red: Yuhactal Variety (Eznab Complex) . . . . . . . . . . . . . . . . . . . 357

Yuhactal Black-on-red: Yuhactal Variety (Imix Complex) . . . . . . . . . . . . . . . . . . . . 313

Zacatel Cream Polychrome: Red-bar Variety . . . . . . . . . . . . . . . . . . . . . 312

Zacatel Cream Polychrome: Zacatel Variety (Eznab Complex) . . . . . . . . . . . . . . . . . 354

Zacatel Cream Polychrome: Zacatel Variety (Imix Complex) . . . . . . . . . . . . . . . . . 311

Zacatel Cream Polychrome: Zacpech Variety . . . . . . . . . . . . . . . . 355 
Zacec Black: Zacec Variety (Ik Complex) . . . . . . . . . . . . . . . . . . . . . . . . . . . 269

Zacec Black: Zacec Variety (Imix Complex) . . . . . . . . . . . . . . . . . . . . . 307 
